\title{
TORSION UNITS IN ALTERNATIVE LOOP RINGS
}

\author{
EDGAR G. GOODAIRE AND CÉSAR POLCINO MILIES
}

(Communicated by Donald S. Passman)

\begin{abstract}
Let $\mathbf{Z} L$ denote the integral alternative loop ring of a finite loop $L$. If $L$ is an abelian group, a well-known result of G. Higman says that $\pm g, g \in L$ are the only torsion units (invertible elements of finite order) in $\mathbf{Z} L$. When $L$ is not abelian, another obvious source of units is the set $\pm \gamma^{-1} g \gamma$ of conjugates of elements of $L$ by invertible elements in the rational loop algebra $\mathbf{Q} L$. H. Zassenhaus has conjectured that all the torsion units in an integral group ring are of this form. In the alternative but not associative case, one can form potentially more torsion units by considering conjugates of conjugates $\gamma_{1}^{-1}\left(\gamma_{2}^{-1} g \gamma_{2}\right) \gamma_{1}$ and so forth. In this paper we prove that every torsion unit in an alternative loop ring over $\mathbf{Z}$ is \pm a conjugate of a conjugate of a loop element.
\end{abstract}

\section{INTRODUCTION}

Let $G$ be a finite group and let $\mathbf{Z} G$ denote the group ring of $\mathrm{G}$ over the ring $\mathbf{Z}$ of rational integers. As usual, we shall denote by $\epsilon: \mathbf{Z} G \rightarrow \mathbf{Z}$ the augmentation map; i.e. the map given by $\epsilon\left(\sum \alpha_{g} g\right)=\sum \alpha_{g}$. If we denote by $U(\mathbf{Z} G)$ the group of units of $\mathbf{Z} G$, then the set $V(\mathbf{Z} G)=\{\alpha \in U(\mathbf{Z} G) \mid \epsilon(\alpha)=1\}$ is called the group of normalized units of $\mathbf{Z} G$. The elements of the form $\pm g, g \in G$ are the trivial units of $\mathbf{Z} G$.

G. Higman, in a classical paper on the units of group rings [7] has shown that if $G$ is abelian, then all units of finite order in $Z G$ are trivial. When $G$ is not abelian, an obvious way to exhibit new torsion units in $\mathbf{Z} G$ is to compute conjugates of the form $\gamma^{-1} g \gamma$, with $g \in G$ and $\gamma \in V(\mathbf{Z} G)$. One can also allow $\gamma$ to belong to $\mathbf{Q} G$ provided that $\gamma^{-1} g \gamma \in \mathbf{Z} G$. H. Zassenhaus has conjectured that all torsion units in $\mathbf{Z} G$ can be constructed in this way. More precisely, we have

Zassenhaus Conjecture. Let $r \in \mathbf{Z} G$ be a normalized unit of finite order. Then there exists an invertible element $\alpha \in \mathbf{Q} G$ and an element $g \in G$ such that $\alpha^{-1} r \alpha=g$.

Received by the editors July 26,1988 .

1980 Mathematics Subject Classification (1985 Revision). Primary 17D05; Secondary 16A26.

Key words and phrases. Alternative ring, group ring, unit.

This work was partially supported by FAPESP (Brasil) and by NSERC (Canada) Grant No. A9087. 
It has been shown that the conjecture holds for some families of groups (see for example [9]), but in general, it remains very much open.

Nonassociative generalizations of group rings have been considered in recent literature [3 and 11]. One defines the loop ring $R L$ of a finite loop $L$ over an associative ring $R$ in precisely the same way a group ring is defined. Since alternative rings resemble associative rings in many ways, it is natural to hope that certain conjectures and properties of associative rings may hold for alternative rings. In this paper, we establish the following alternative analogue of the Zassenhaus conjecture.

0.1 Theorem. Let $r$ be a normalized torsion unit in the integral alternative loop ring $\mathbf{Z} L$ of a finite loop $L$. Then there exist units $\gamma_{1}, \gamma_{2} \in \mathbf{Q} L$ and $l \in L$ such that $\gamma_{2}^{-1}\left(\gamma_{1}^{-1} r \gamma_{1}\right) \gamma_{2}=l$.

(Notice that this theorem gives a statement of the Zassenhaus Conjecture for the variety of alternative rings which reduces to the usual conjecture in the subvariety of associative rings. The import of this paper, then, is to show that the extended conjecture is at least true for those alternative loop rings which are not associative.)

\section{SOME BASIC FACTS}

An $R A$ loop is a loop whose loop rings over any ring of characteristic $\neq 2$ are alternative but not associative. We list a few facts about $R A$ loops which can be found in [5].

1.1 Proposition. Let $L$ be an $R A$ loop. Then

1. the nucleus and centre of $L$ coincide;

2. the commutator subloop $L^{\prime}$ is generated by a central element $e$ of order 2 ;

3. there exists a non-abelian group $G \subset L$ and an element $u \in L$ such that $L=G \cup G u$. The centre of $G$ coincides with the centre of $L$ and shall be denoted by $\mathscr{Z}$. For every element $l \in L$ we have $l^{2} \in \mathscr{Z}$;

4. the $\operatorname{map}^{*}: L \rightarrow L$ given by

$$
g^{*}= \begin{cases}g & \text { if } g \in \mathscr{Z} \\ e g & \text { if } g \notin \mathscr{Z}\end{cases}
$$

is an involution of $L$ which extends linearly to $R L$;

5. every element $r \in R L$ can be written uniquely in the form $r=x+y u$ with $x, y \in R G$. Setting $g_{0}=u^{2}$, the involution and multiplication in $R L$ are given respectively by

$$
\begin{aligned}
(x+y u)^{*} & =x^{*}+e y u \\
(x+y u)(z+w u) & =\left(x z+g_{0} w^{*} y\right)+\left(w x+y z^{*}\right) u .
\end{aligned}
$$


Letting $\mathscr{Z}(R G)$ denote the centre of the group ring $R G$, it is easy to see that the centre of $R L$ is

$$
\mathscr{Z}(R L)=\{x+y u \mid x, y \in \mathscr{Z}(R G), y=e y\} .
$$

It follows that $r \in \mathscr{Z}(R L)$ if and only if $r=r^{*}$; in particular, $r+r^{*}$ and $r r^{*}$ are central for any $r \in R L$.

Now we turn our attention to augmentation ideals. Let $N$ be a normal subloop of an RA loop $L$. Just as in the case of group rings, the natural map $L \rightarrow L / N$ can be extended to a ring homomorphism $\omega: \mathbf{Z} L \rightarrow \mathbf{Z}(L / N)$. It is easily shown that $\operatorname{ker}(\omega)$ is spanned over $\mathbf{Z} L$ by the elements of the set $\{n-1 \mid n \in N\}$. In the case where $L$ is a group $G$, we denote the kernel of $\omega$ by $\Delta(G, N)$ and write $\Delta(G)$ rather than $\Delta(G, G)$; this ideal is precisely the kernel of the augmentation map $\epsilon$ and is called the augmentation ideal of Z $G$. If $N \subseteq \mathscr{Z}$, then clearly $N^{*}=N$. On the other hand, if $N$ contains a noncentral element $n$, then there exists $l \in L$ such that $l^{-1} n l=e n \in N$ and hence $e \in N$. It follows that $N^{*} \subseteq N$ always holds.

Now write $L=G \cup G u$ with $G$ as in Proposition 1.1 and assume that $N \subseteq G$. Let $x+y u$ be any element in $\mathbf{Z} L$ with $x, y \in \mathbf{Z} G$ and let $n \in N$. Then

$$
(x+y u)(n-1)=x(n-1)+y(n-1)^{*} u \in \Delta(G, N)+\Delta(G, N) u .
$$

These remarks show

1.2 Proposition. With the notation as above,

1. an element $x+y u \in \mathbf{Z} L$ belongs to $k e r(\omega)$ if and only if $x, y \in$ $\Delta(G, N)$

2. $\Delta(G, N)^{*} \subseteq \Delta(G, N)$.

We require additional properties of augmentation ideals. The identity $x=$ $\epsilon(x)+(x-\epsilon(x))$ for $x \in \mathbf{Z}(G)$ implies that $\mathbf{Z} G=\mathbf{Z}+\Delta(G)$. Hence, for any $N \triangleleft G, \Delta(G, N)=\mathbf{Z} G \Delta(N)=\Delta(N)+\Delta(G) \Delta(N)$, from which it follows that

$$
[\Delta(G, N)]^{2} \subseteq \Delta(G) \Delta(N) \subseteq \Delta(G, N) .
$$

In [8], Karpilovsky gives a remarkably short proof of the following important result of Whitcomb:

1.3 Lemma (Whitcomb). Let $x \in \mathbf{Z} G$ and suppose that $x$ can be written in the form $x=g^{\prime}+\delta^{\prime}$ for some $g^{\prime} \in G$ and $\delta^{\prime} \in \Delta(G, N)$. Then there exist $g \in G$ and $\delta \in \Delta(G) \Delta(N)$ such that $x=g+\delta$.

In the same article, Karpilovsky proves that $G \cap(1+\Delta(G) \Delta(N))=N^{\prime}$ (the commutator subgroup of $N)$. We shall need the following implication of this fact:

$$
\text { If } N \triangleleft G \text { is abelian, } G \cap(1+\Delta(G) \Delta(N))=1 \text {. }
$$

It is in the case $N=G^{\prime}$ that these results will be used (and usually implicity) in the sequel. 
We conclude this section with the remark that the augmentation map on $\mathbf{Z} G$ has a natural extension to $\mathbf{Z} L$. This extended map, which we continue to denote $\epsilon$ and to which we continue to refer as the augmentation map, is a ring homomorphism.

\section{TORSION UNITS}

In this section, we note that a well-known property of torsion units in group rings also holds in alternative loop rings and then derive consequences peculiar to the nonassociative situation. Throughout the rest of this paper, $L$ will always denote a finite RA loop.

2.1 Proposition. Let $r=\sum_{l \in L} \alpha_{l} l$ be a unit of finite order in the integral loop ring of $L$. If $\alpha_{1} \neq 0$, then $r=\alpha_{1}= \pm 1$.

Proof. For a given element $r=\sum \alpha_{l} l \in \mathbf{Z} L$, the map $R_{r}: \mathbf{Z} L \rightarrow \mathbf{Z} L$ defined by $R_{r}(t)=t r$ for $t \in \mathbf{Z L}$ is linear and satisfies $R_{r^{m}}=R_{r}^{m}$ because in an alternative ring, the subring generated by any pair of elements, for example $t$ and $r$, is associative. (We use Schafer's book [13] as our basic reference for alternative rings.) Following Berman [1], we note that the matrix for $R_{r}$ is similar over C to a diagonal matrix $A=\operatorname{diag}\left(\xi_{1}, \ldots, \xi_{n}\right)$, the $\xi_{i} m$ th roots of unity for some $m$. Thus the trace of $R_{r}$ is on the one hand $\sum_{i=1}^{n} \xi_{i}$ while on the other it is $\sum \alpha_{l} \operatorname{tr}\left(R_{l}\right)=n \alpha_{1}$. Thus all the $\xi_{i}$ are equal, and in fact equal to $\alpha_{1}$, and $\alpha_{l}=0$ if $l \neq 1$.

2.2 Corollary. If $r=\sum \alpha_{l} l \in L$ is a torsion unit in $\mathbf{Z} L$ and $\alpha_{l} \neq 0$ for some $l \in \mathscr{Z}$, then $r= \pm l$.

Proof. The element $l^{-1} r$ is a torsion unit with nonzero coefficient of 1 .

2.3 Corollary. Let $r$ be a torsion unit in $\mathrm{ZL}$. Then $r^{2} \in \mathscr{Z}$.

Proof. Let $\mathrm{S}$ denote the support of $r$; i.e. $r=\sum_{l \in S} \alpha_{l} l$ with $\alpha_{l} \neq 0$ for all $l \in S$. Then we can write

$$
r^{2}=\sum_{l \in S} \alpha_{l}^{2} l^{2}+\beta_{1}+\beta_{2}
$$

where $\beta_{1}=2 \sum \alpha_{l} \alpha_{m} l m$, the sum running over all pairs $(l, m) \in L \times L$ such that $l m=m l$ and $\beta_{2}=\sum \alpha_{l} \alpha_{m}(l m+m l)$, the sum running over all pairs $(l, m)$ such that $l m \neq m l$. Now both $\epsilon\left(\beta_{1}\right)$ and $\epsilon\left(\beta_{2}\right)$ are even integers while $\epsilon\left(r^{2}\right)=1$ since $r$ is a unit. Therefore $\alpha_{l_{1}}$ must be odd for some $l_{1} \in S$. Since $l_{1}^{2}$ is central, it cannot be equal to an element $l m$ in the support of $\beta_{2}(l \mathrm{~m}$ central $\Longrightarrow l m=m l)$; moreover, the term $\alpha_{l_{1}}^{2} l_{1}^{2}$ cannot cancel with a term of the form $2 \alpha_{l} \alpha_{m} l m$. Hence $l_{1}^{2} \in \operatorname{supp}\left(r^{2}\right)$ and now Corollary 2.2 shows that $r^{2}= \pm l_{1}^{2}$. Finally, since $\epsilon\left(r^{2}\right)=1$, we must have $r^{2}=l_{1}^{2} \in \mathscr{Z}$.

Notice that this proof actually shows that $r^{2}$ is equal to the square of an element in $L$. We wish to determine this element in a precise way. 
Let $\omega: \mathbf{Z} L \rightarrow \mathbf{Z}\left(L / L^{\prime}\right)$ denote the homomorphism induced by the natural map $L \rightarrow L / L^{\prime}$ and let $r \in V(\mathbf{Z} G)$ have finite order. Then $\omega(r)$ is a unit of finite order in the integral abelian group ring $\mathbf{Z}\left(L / L^{\prime}\right)$ and is thus trivial [14, Corollary II.1.8]. Hence either $\omega(r)=\omega\left(g^{\prime}\right)$ or $\omega(r)=\omega\left(g^{\prime} u\right)$ for some $g^{\prime} \in G$. If $\omega(r)=\omega\left(g^{\prime}\right)$, we can write $r$ in the form

$$
r=g^{\prime}+\delta+\delta_{2} u, \quad \text { where } \delta, \delta_{2} \in \Delta\left(G, G^{\prime}\right) .
$$

By Whitcomb's Lemma, we can write $g^{\prime}+\delta=g+\delta_{1}$ for some $g \in G$ and some $\delta_{1} \in \Delta(G) \Delta\left(G^{\prime}\right), \Delta\left(G^{\prime}\right)$ the augmentation ideal of $\mathbf{Z} G^{\prime}$. Consequently, we can write $r$ in the form

$$
r=g+\delta_{1}+\delta_{2} u, \quad \delta_{1} \in \Delta(G) \Delta\left(G^{\prime}\right), \delta_{2} \in \Delta\left(G, G^{\prime}\right) .
$$

In a similar way, if $\omega(r)=\omega\left(g^{\prime} u\right)$, we can write $r$ in the form

$$
r=\left(g+\delta_{1}\right) u+\delta_{2}, \quad \delta_{1} \in \Delta(G) \Delta\left(G^{\prime}\right), \delta_{2} \in \Delta\left(G, G^{\prime}\right) .
$$

2.4 Proposition. Let $r$ be a normalized unit of finite order in $\mathbf{Z} L$. Then $r^{2}=g^{2}$ or $r^{2}=(g u)^{2}$ according as $r$ is of the form (2) or (3) respectively.

Proof. Assume first that $r$ can be written in the form $r=g+\delta_{1}+\delta_{2} u$ as in (2). Then it is easy to see that $r^{2}$ can be written as

$$
r^{2}=g^{2}+\delta_{1}^{\prime}+\delta_{2}^{\prime} u, \quad \delta_{1}^{\prime} \in \Delta(G) \Delta\left(G^{\prime}\right), \delta_{2}^{\prime} \in \Delta\left(G, G^{\prime}\right) .
$$

We know from Corollary 2.3 that $r^{2} \in \mathscr{Z} \subseteq G$, so we must have $r^{2}=g^{2}+\delta_{1}^{\prime}$ and $\delta_{2}^{\prime} u=0$. Hence, using (1),

$$
g^{-2} r^{2}=1+\delta_{2}^{\prime \prime} \in G \cap\left(1+\Delta(G) \Delta\left(G^{\prime}\right)\right)=1
$$

and so $r^{2}=g^{2}$. In a similar way it can be shown that if $r=\left(g+\delta_{1}\right) u+\delta_{2}$ as in (3), then $r^{2}=(g u)^{2}$.

In the remainder of this paper, we prove Theorem 0.1 by showing that a normalized torsion unit $r \in \mathbf{Z} L$ is conjugate in $\mathbf{Q} L$ to a conjugate of the element $g$ or $g u$ found above. J. Ritter and S. K. Sehgal have shown that something similar happens when working with integral group rings of nilpotent class 2 groups and have furthermore shown that this need not be the case in general [12].

\section{A REDUCTION}

C. Polcino Milies and S. K. Sehgal have shown that the Zassenhaus Conjecture for group rings can be reduced to the question of conjugacy in the complex group algebra $\mathbf{C} G$ [10]. Now an old, but perhaps not well-known, theorem of R. H. Bruck [2, Theorem 7A] says that over a field of characteristic 0 , the loop algebra of any finite loop is semisimple. Thus, if $L$ is a finite RA loop, the alternative loop algebra $\mathbf{C} L$ is the direct sum of simple alternative algebras which, in turn, are known to be either simple associative or Cayley-Dickson 
algebras. In both such types of algebra an element is either invertible or a zero divisor, in the Cayley-Dickson case because $a$ is invertible if and only if $a \bar{a} \neq 0$ ( $\bar{a}$ denoting the image of $a$ under the canonical involution). The argument of Ritter and Sehgal presented in [10, Lemma 5] now works verbatim to establish

3.1 Lemma. Let $k \subseteq K$ be infinite fields. Let $L$ be a finite loop whose loop algebra over $k$ is semisimple and alternative. Then if two elements $\alpha, \beta \in k L$ are conjugate in $K L$, they are also conjugate in $k L$.

As an immediate consequence, we obtain

3.2 Corollary. If $\alpha, \beta, \gamma \in \mathbf{Q} L$ and $\alpha$ and $\beta$ are conjugate to $\gamma$ in $\mathbf{C} L$, then $\alpha$ and $\beta$ are conjugate to $\gamma$ in $\mathbf{Q} L$.

Because of this result, we shall work in $\mathbf{C} L$ throughout the rest of this paper. Write $\mathbf{C} L=A_{1} \oplus \cdots \oplus A_{n}$ where the $A_{i}$ are simple alternative algebras. Since $A_{i} \cap A_{i}^{*}$ is a nonzero ideal contained in $A_{i}$, it follows that $A_{i}^{*}=A_{i}$ for each i. Given $r \in \mathbf{C} L$, we shall denote by $r_{i}$ the component of $r$ in $A_{i}$. To prove that $r$ and $s \in \mathbf{Q} L$ have a common conjugate in $\mathbf{C} L$, it suffices to show that their respective components $r_{i}$ and $s_{i}$ have a common conjugate in $A_{i}$, for all $i$.

We define a trace-like map $\theta: \mathbf{C} L \rightarrow \mathbf{C} L$ by $\theta(r)=r+r^{*}$. Notice that $\theta$ is a linear map whose values lie in the centre of $C L$. Also, $\theta(r)=\theta\left(r^{*}\right)$ for any $r \in \mathbf{C} L$ and, if $r \in \mathbf{C} L$ is central, then $\theta(r)=2 r$. Since $A_{i}^{*}=A_{i}$, each simple component of $\mathbf{C} L$ is invariant under $\theta$.

3.3 Lemma. Let $\delta \in \Delta\left(G, G^{\prime}\right)$. Then $\theta\left(\delta_{i} u_{i}\right)=0$ and, if $\operatorname{supp}(\delta) \cap \mathscr{Z}=\varnothing$, then also $\theta\left(\delta_{i}\right)=0$.

Proof. First, since e is central, its component $e_{i}$ in $A_{i}$ lies in the centre $\mathscr{Z}\left(A_{i}\right) \cong \mathrm{C}$. Since $e^{2}=1$, we have $e_{i}= \pm 1$ in $A_{i}$. Now an element $\delta \in$ $\Delta\left(G, G^{\prime}\right)$ is of the form $\delta=\alpha(1-e)$ for some $\alpha$ in $\mathbf{C} G$. If $e_{i}=1$, it is clear that $\theta\left(\delta_{i} u_{i}\right)=\theta\left(\delta_{i}\right)=0$, so we assume henceforth that $e_{i}=-1$. On the one hand we have $\theta\left(\left(\delta_{i} u_{i}\right)^{*}\right)=\theta\left(\delta_{i} u_{i}\right)$ while on the other, since $\left(\delta_{i} u_{i}\right)^{*}=e_{i} \delta_{i} u_{i}$, we get $\theta\left(\left(\delta_{i} u_{i}\right)^{*}\right)=-\theta\left(\delta_{i} u_{i}\right)$. Thus $\theta\left(\delta_{i} u_{i}\right)=0$.

Now assume that $\operatorname{supp}(\delta) \cap \mathscr{Z}=\varnothing$. Writing $\delta=\sum \alpha_{g} g(1-e)$, it is easy to see that $\alpha_{g}=0$ for every $g \in \mathscr{Z}$. Now when $g$ is not central, $\theta\left(g_{i}\right)=$ $\theta\left(g_{i}^{*}\right)=\theta\left(e_{i} g_{i}\right)=-\theta\left(g_{i}\right)$ and so $\theta\left(g_{i}\right)=0$. Thus $\theta\left(\delta_{i}\right)=2 \sum \alpha_{g} \theta\left(g_{i}\right)=0$, as claimed.

3.4 Lemma. Let $r$ and $s$ be units in $C L$ such that $\operatorname{supp}(r) \cap \mathscr{Z}=\operatorname{supp}(s) \cap \mathscr{Z}=$ $\varnothing$. Then $r$ is central if and only if $s$ is central; $r_{i}$ is central if and only if $s_{i}$ is central; and if $r_{i}$ is central and $\theta\left(r_{i}\right)=\theta\left(s_{i}\right)$, then $r_{i}=s_{i}$.

Proof. Our hypothesis on the supports of $r$ and $s$ implies that $r^{*}=e r$ and $s^{*}=e s$; hence $(r s+s r)^{*}=e^{2}(r s+s r)=r s+s r$. Thus $r s+s r$ is central. Since $r$ and $s$ are units, $r$ is certainly central if and only if $s$ is central. A similar statement holds for $r_{i}$ and $s_{i}$ because $r s+s r$ central implies $r_{i} s_{i}+s_{i} r_{i}$ 
central. Finally, if $r_{i}$ is central, then $\theta\left(r_{i}\right)=2 r_{i}$. But also $s_{i}$ is central and so $\theta\left(s_{i}\right)=2 s_{i}$. The last statement of the Lemma now follows.

\section{THE CONJECTURE}

We are now ready to prove Theorem 0.1 . Let $r$ therefore denote a normalized torsion unit in the integral alternative loop ring of a finite loop $L$. We shall prove that there exists an $l \in L$ such that $r$ and $l$ are conjugate in $\mathbf{Q} L$ to the same element (of $\mathbf{Q} L$ ). If $\operatorname{supp}(r) \cap \mathscr{Z} \neq \varnothing$ then Corollary 2.2 shows that $r=l$ for some central $l \in L$. So we assume henceforth that $\operatorname{supp}(r) \cap \mathscr{Z}=\varnothing$. From the preamble to Proposition 2.4, we see that $r$ can be written either in the form

$$
r=g+\delta_{1}+\delta_{2} u \text { or } \quad r=g u+\delta_{1} u+\delta_{2}
$$

with $\delta_{1} \in \Delta(G) \Delta\left(G^{\prime}\right)$ and $\delta_{2} \in \Delta\left(G, G^{\prime}\right)$. We consider these two cases separately.

Case 1. Assume $r=g+\delta_{1}+\delta_{2} u$. Then $r^{2}=g^{2}$ by Proposition 2.4. Since $\operatorname{supp}(r) \cap \mathscr{Z}=\varnothing$, it follows that $g \notin \mathscr{Z}$ and $\operatorname{supp}\left(\delta_{1}\right) \cap \mathscr{Z}=\varnothing$. By Lemma 3.3, $\theta\left(r_{i}\right)=\theta\left(g_{i}\right), i=1, \ldots, n$. Lemma 3.4 then shows that we should study only those components where $r_{i}$ and $g_{i}$ are noncentral. Moreover we assume $e_{i}=-1$ because if $e_{i}=+1$, then $\left(\delta_{1}\right)_{i}=\left(\delta_{2}\right)_{i}=0$ and $r_{i}=g_{i}$.

Henceforth, we shall work inside a fixed component $A_{i}$ (where $e_{i}=-1$ and $r_{i}, g_{i}$ are noncentral) and shall omit the subscript $i$ for clarity. Let $B$ be the (associative) subalgebra of $A$ generated by $r$ and $g$. Since the centre of a simple finite dimensional algebra over $\mathbf{C}$ is $\mathbf{C}$ and since $r^{2}=g^{2}$ and $r g+g r$ are central, we can write $r^{2}=g^{2}=\lambda^{2}, r g+g r=\mu, \lambda, \mu \in \mathbf{C}$. Every element in $B$ is a linear combination of $1, r, g$ and $r g$. Set $f_{1}=\frac{1}{2}(1+r / \lambda)$ and $f_{2}=\frac{1}{2}(1-r / \lambda)$. Then $f_{1}$ and $f_{2}$ are orthogonal idempotents $\neq 0,1$ such that $f_{1}+f_{2}=1$ and $r=\lambda\left(f_{1}-f_{2}\right)$. Thus $B=\left(f_{1}+f_{2}\right) B\left(f_{1}+f_{2}\right)=\sum f_{i} B f_{j}$. Defining $f_{i j}=f_{i} g f_{j}$ we have by straightforward computation,

$$
\begin{gathered}
f_{11}=\frac{\mu}{2 \lambda} f_{1}, \quad f_{22}=-\frac{\mu}{2 \lambda} f_{2} \\
f_{12}=f_{1} g-\frac{\mu}{2 \lambda} f_{1}, \quad \text { and } f_{21}=f_{2} g+\frac{\mu}{2 \lambda} f_{2} .
\end{gathered}
$$

It follows that $f_{i} B f_{i}$ is one-dimensional with basis $f_{i}, i=1,2$, and $\operatorname{dim}\left(f_{i} B f_{j}\right) \leq 1$ with equality provided $f_{i j} \neq 0$, in which case $f_{i} B f_{j}$ has basis $f_{i j}$. Moreover,

$$
f_{12}^{2}=f_{21}^{2}=0, \quad f_{12} f_{21}=\left(\lambda^{2}-\frac{\mu^{2}}{4 \lambda^{2}}\right) f_{1} \quad \text { and } \quad f_{21} f_{12}=\left(\lambda^{2}-\frac{\mu^{2}}{4 \lambda^{2}}\right) f_{2} .
$$

If $\lambda^{2}-\mu^{2} / 4 \lambda^{2} \neq 0$ it is apparent that $B \cong M_{2}(\mathbf{C})$. Since the minimal polynomial of both $r$ and $g$ over $\mathbf{C}$ is $X^{2}-\lambda^{2}$, each element is similar to $\left(\begin{array}{cc}\lambda & 0 \\ 0 & -\lambda\end{array}\right)$, so $r$ and $g$ are both conjugate to $g$ in $B$, hence in $A_{i}$. If $\lambda^{2}-\mu^{2} / 4 \lambda^{2}=0$, then $\mu / 2 \lambda^{2}= \pm 1$. 
Assume first that $\mu / 2 \lambda^{2}=1$. Defining $s=(1 / 2 \lambda)(g+r)$, it can be checked directly that $s^{2}=1$ and $s^{-1} r s=g$. Again then, $r$ and $g$ are conjugate to the element $g$ in $A_{i}$.

If $\mu / 2 \lambda^{2}=-1$, define $t=(1 / 2 \lambda)(g-r)$ and check that $t^{2}=1$ and $t^{-1} r t=$ $-g$. Since $g$ is not central, for some $h$ in the projection of $\mathbf{C} L$ in $A_{i}$, we must have $h^{-1} g h=e g=-g$, so it follows here too that $r$ and $g$ have a common conjugate in $A_{i}$, although this time, this conjugate is $-g$.

Case 2. Assume now that $r=g u+\delta_{1} u+\delta_{2}$ and hence $r^{2}=(g u)^{2}$. Since $\operatorname{supp}(r) \cap \mathscr{Z}=\varnothing$, we easily see that $\operatorname{supp}\left(\delta_{2}\right) \cap \mathscr{Z}=\varnothing$ and hence $\theta\left(r_{i}\right)=$ $\theta\left(g_{i} u_{i}\right)$. Once again then, we may suppose that $r_{i}$ and $g_{i} u_{i}$ are not central in $A_{i}$ and that $e_{i}=-1$. Now we essentially repeat the argument of Case 1 to obtain the desired conclusion.

In both cases, $r$ and $l=g$ or $g u$ have a common conjugate in $C L$. Corollary 3.2 then gives the desired result.

\section{ACKNOWLEDGMENT}

The authors thank the referee especially for encouraging us to amplify certain parts of this paper and thereby (we hope) make it accessible to a wider readership.

\section{REFERENCES}

1. S. D. Berman, On the equation $x^{m}=1$ in an integral group ring, Ukrain. Mat. $\mathrm{Zh} .7$ (1955), 253-261.

2. Richard H. Bruck, Some results in the theory of linear non-associative algebras, Trans. Amer. Math. Soc. 56 (1944), 141-199.

3. Orin Chein and Edgar G. Goodaire, Loops whose loop rings are alternative, Comm. Algebra 14 (1986), 293-310.

4. E. G. Goodaire and M. M. Parmenter, Units in alternative loop rings, Israel J. Math. 53 (1986), 209-216.

5. E. G. Goodaire and M. M. Parmenter, Semi-simplicity of alternative loop rings, Acta Math. Hungary 50 (1987), 241-247.

6. Edgar G. Goodaire and César Polcino Milies, Isomorphisms of integral alternative loop rings, Rend. Circ. Mat. Palermo (2) (to appear).

7. G. Higman, The units of group rings, Proc. London Math. Soc. 246 (1940), 231-248.

8. G. Karpilovsky, On the isomorphism problem for integral group rings, J. Algebra 59 (1979), $1-4$.

9. C. Polcino Milies, Torsion units in group rings and a conjecture of H. J. Zassenhaus, Group and semigroup rings, G. Karpilovsky (Ed.), North Holland Math. Studies No. 126, Elsevier, Amsterdam, 1986, 179-192.

10. C. Polcino Milies and S. K. Sehgal, Torsion units in integral group rings of metacyclic groups, J. Number Theory 19 (1984), 103-114.

11. J. Marshall Osborn, Lie-admissible noncommutative Jordan loop rings, Algebras Groups Geom. 1 (1984), 435-489.

12. J. Ritter and S. K. Sehgal, On a conjecture of Zassenhaus on torsion units in integral group rings, Math. Ann. 264 (1983), 257-270. 
13. R. D. Schafer, An introduction to nonassociative algebras, Academic Press, New York, 1966. 14. S. K. Sehgal, Topics in group rings, Marcel Dekker, New York, 1977.

Department of Mathematics \& Statistics, Memorial University of Newfoundland, St. John's, Newfoundland, Canada A1C 5S7

Instituto de Matemática e Estatística, Universidade de São Paulo, Caixa Postal 20570 Ag. Iguatemi, São Paulo, Brazil 